\title{
Comparison of pre- and post-processors for ensemble streamflow prediction
}

\author{
Tae-Ho Kang,' Young-Oh Kim²* and II-Pyo Hong' \\ ' Water Resources Research Division, Korea Institute of Construction Technology, I 190, Siminidae-Ro, Ilsanseo-Gu, Goyang-Si, Gyeonggi-Do, 4 I I-7 I 2, \\ Republic of Korea \\ ${ }^{2}$ Department of Civil and Environmental Engineering, Seoul National University, 599 Gwanak-Ro, Gwanak-Gu, Seoul I 5 I-742, Republic of Korea
}

* Correspondence to:

Young-Oh Kim, Department of Civil and Environmental

Engineering, Seoul National University, 599 Gwanak-Ro,

Gwanak-Gu, Seoul 151-742,

Republic of Korea.

E-mail: yokim05@snu.ac.kr

Received: 22 September 2009

Revised: 14 March 2010

Accepted: 15 March 2010

\begin{abstract}
This study conducted a broad review of the pre- and post-processor methods for ensemble streamflow prediction using a Korean case study. Categorical forecasts offered by the Korea Meteorogical Administration and deterministic forecasts of a regional climate model called Seoul National University Regional Climate Model(SNURCM) were selected as climate forecast information for the pre-processors and incorporated into Ensemble Streamflow Prediction(ESP) runs with the TANK hydrologic model. The post-processors were then used to minimize a possible error propagated through the streamflow generation. The application results show that use of the post-processor more effectively reduced the uncertainty of the no-processor ESP than use of the pre-processor, especially in dry season. Copyright $\subset 2010$ Royal Meteorological Society
\end{abstract}

Keywords: ensemble streamflow prediction; probabilistic climate forecast; hydrologic model; pre-processor; post-processor; uncertainty

\section{Introduction}

One of the prevalant methdologies of probabilistic streamflow prediction is ensemble streamflow prediction (ESP). Day (1985) examined the theory, capabilities and potential applications of the ESP procedure using the National Weather Service (NWS) River Forecast System, and the NWS has used ESP mainly for long-range streamflow prediction. Although the basic theory of ESP is simple, there has been a considerable amount of research addressing the uncertainty of ESP. Many studies have attempted to reduce the considerable uncertainty of the meteorological inputs of ESP because it is difficult to obtain meaningful results from ESP with raw meteorological inputs. The methodologies that can reduce this meterorlogical input uncertainty are categorized as pre-processor methods in this study. However, uncertainties in the hydrologic model structure, parameters and initial conditions have also been studied because such uncertainties could produce biased ESP results (Seo et al., 2006). Shi et al. (2008) proved that the bias correction method reduces the bias from the hydrologic model, whereas the model calibration reduces not only the bias but also the spread of the ESP hindcast ensemble means. The methodologies that reduce the hydrologic model uncertainties are called the postprocessor methods of an ESP system in this study. This study compiled a broad review of these preand post-processor methodologies, compared their features, and tested their performances using case studies in Korea.

\section{Methodology}

If the available climate forecast is not probabilistic, an additional procedure is required to transform the nonprobabilistic climate forecast into a probabilistic forecast. This procedure is referred to as Pre-processor Step 1. Pre-processor Step 2 then uses the probabilistic forecast to adjust the meteorological ensembles. Conversely, the post-processor reduces hydrological uncertainties caused by basin initial conditions, model parameters and model structures (Figure 1).

\section{I. The pre-processor step I}

2.I.I. Conversion from categorical to probabilistic forecasts

Kang et al. (2007) proposed a simple method to produce a probabilistic forecast from a categorical forecast that calculates a most probable interval. The below-normal, normal and above-normal categories correspond to ranges below 0.7 , between 0.7 and 1.2 , and above 1.2 times the last 30-year average of monthly meteorological variables (e.g. temperature and precipitation), respectively.

In this study, we call the method proposed by Kang et al. (2007) the Categorical to the Probabilistic forecasts Conversion (CPC). To implement CPC, the entire historical observed data set of the forecasted variables in this study is divided into three subsets based on the category of the historical KMA forecasts. For the above-normal category, for example, only the observed data from the months when the above-normal 


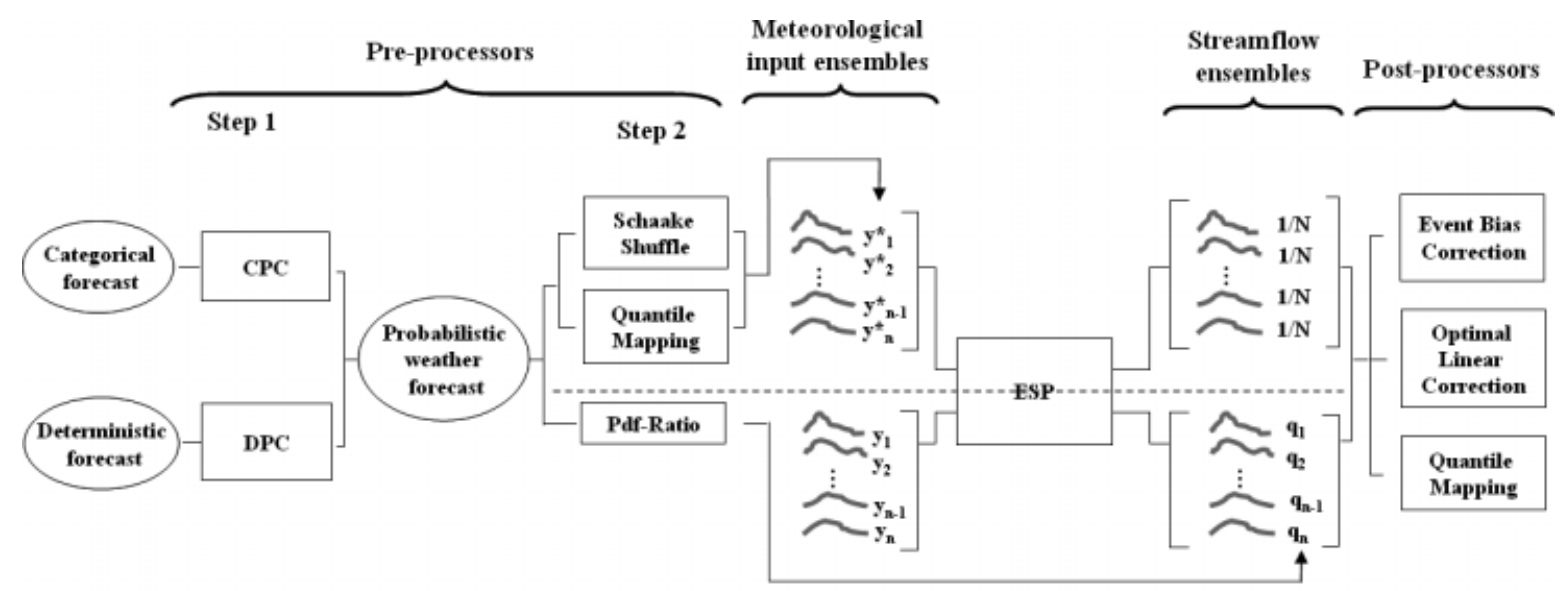

y : An original meteorological ensemble member $1 / \mathrm{N}$ : Original probability of each ensemble member

$y^{*}$ : A revised meteorological ensemble member $\quad$ q : Revised probability of each ensemble member

Figure I. The ESP procedure with pre- and post-processors.
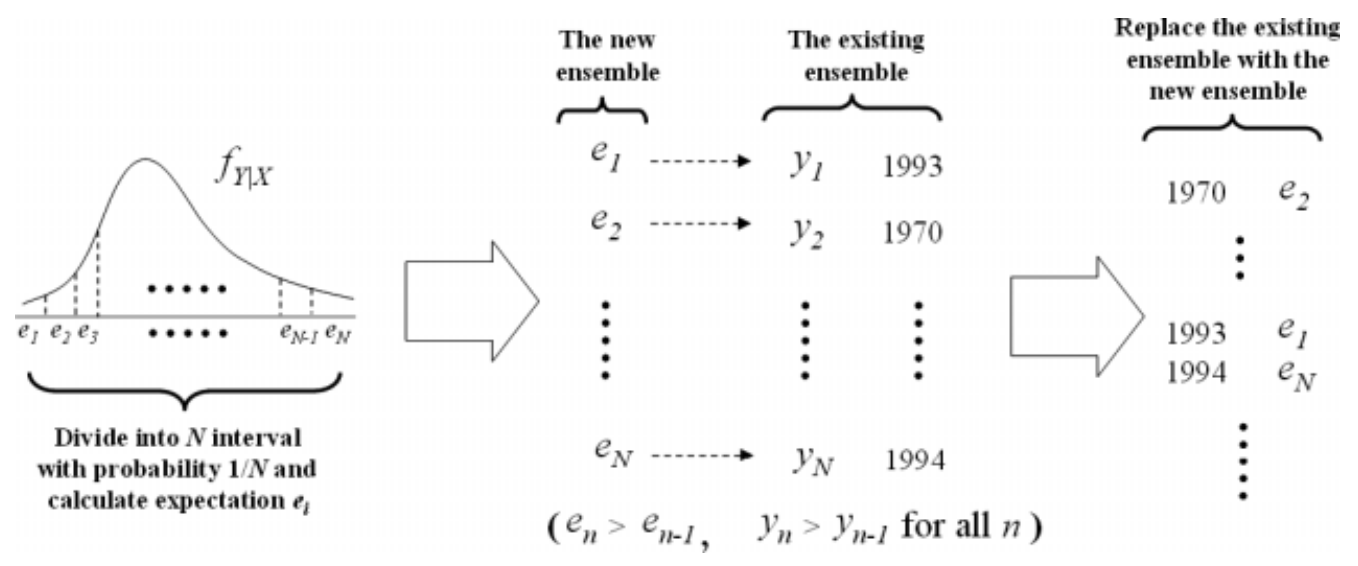

Figure 2. Illustration of the Schaake shuffle method.

category was issued were grouped. The conditional distribution of the forecasted variables is then fitted with the selected subset of the historical data of the forecasted variables.

\section{I.2. Conversion from deterministic to probabilistic forecasts}

Schaake et al. (2007) proposed a method to produce a probabilistic forecast from a deterministic and singlevalue forecast. We call this method the 'Deterministic to Probabilistic forecasts Conversion (DPC)' method. Basically, if the distribution of the observation and the forecast is normal such as the case of temperature, the conditional distribution which is also normal is derived by dividing the joint distribution of the observation $Y$ and the forecast $X$ with a marginal distribution of the forecast $X$ which has the following conditional moments $\mu_{Y \mid X}$ and $\sigma_{Y \mid X}$.

$$
\begin{aligned}
\mu_{Y \mid X} & =\mu_{Y}+\rho_{X Y}\left(\sigma_{Y} / \sigma_{X}\right)\left(x-\mu_{X}\right) \\
\sigma_{Y \mid X} & =\sigma_{Y}^{2}\left(1-\rho_{X Y}^{2}\right)
\end{aligned}
$$

After all, $f_{Y \mid X}$ could be used in the Pre-processor Step 2 as the probabilistic weather forecasts. However, the more complicated methodology including normal transformation is necessary for the case of precipitation as described by Schaake et al. (2007) because precipitation has an intermittent property with the amount being zero and the distribution of precipitation can hardly be expected to be normal. See Schaake et al. (2007) for details.

\subsection{The pre-processor step 2}

\subsection{Schaake shuffle}

The Schaake shuffle (SS) method applied by Clark et al. (2004) and Schaake et al. (2007) partitions the forecast probability distribution $f_{Y \mid X}$ into $N$ intervals where $N$ is the number of scenarios (i.e. the number of years) of a meteorological ensemble. The SS method assigns an equal probability of $1 / N$ to each interval, as shown in Figure 2. New meteorological ensemble values are then constructed by extracting the expected values $\left(e_{n}\right.$ where $n$ represents the $n$th partitioned interval) for all partitioned intervals. The next step is to rank the new ensemble as well as the existing meteorological input ensemble of ESP from the smallest to the largest values. Finally, each of the existing input ensemble members, $y_{n}$, is replaced 


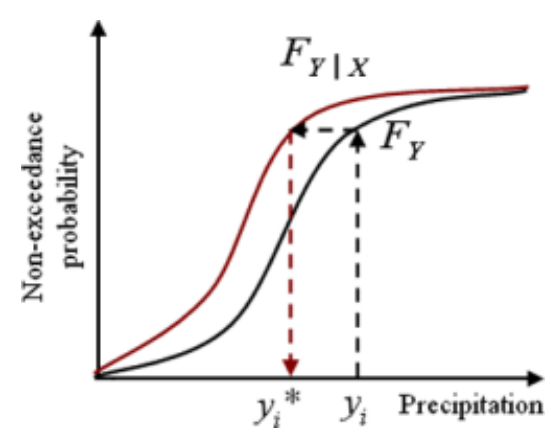

Figure 3. Illustration of the quantile mapping method.

with the new ensemble member, $e_{n}$, that has having the same rank with $y_{n}$ which shares the year number (Figure 2).

\subsubsection{Quantile mapping}

The Quantile mapping (QM) method, as applied by Shi et al. (2008) as the name of 'percentile mapping', is used to reduce the meteorological input uncertainty in this study. QM uses two cumulative distribution functions (CDFs): one is the unconditional $\mathrm{CDF} F_{Y}$ of the observation $Y$ and the other is the conditional CDF $F_{Y \mid X}$ of the observation $Y$ given the climate forecast $X$. The second CDF may be determined during the pre-process step 1. As shown in Figure 3, the existing meteorological ensemble member, $y_{i}$, is adjusted to $y_{i}{ }^{*}$ to make $F_{Y}\left(y_{i}\right)=F_{Y \mid X}\left(y_{i}{ }^{*}\right)$.

\subsubsection{PDF-ratio}

Unlike the SS and QM method, the PDF-ratio revises the probability of each meteorological scenario based on the probabilistic climate forecast. All the input scenarios of ESP initially have the equal probability of $1 / N$ if no information is available. With a climate forecast, the new probability, $q_{i}$, of each meterological input and, at the same time, ensemble streamflow output can be derived as follows (Stedinger and Kim, 2002).

$$
q_{i}=(1 / N)\left\{f_{Y \mid X}(y) / f_{Y}(y)\right\}
$$

where $f_{Y}$ is the initial probability density function (PDF) of the observation $Y$ and $f_{Y \mid X}$ is the updated PDF of the observation $Y$ given the climate forecast $X$.

\subsection{Post-processors}

\subsection{Event bias correction}

Smith et al. (1992) proposed the event bias correction (EBC) method, which corrects the bias of the estimated values using the ratio between true and estimated values. First, the EBC method calculates a ratio for month $m$ and year $i$ that equals $\left(Q^{O}{ }_{i, m} / Q^{S}{ }_{i, m}\right)$, where $m$ is a month when an ensemble forecast is made, $i$ is a year out of $I$ ensemble scenarios, $Q^{O}{ }_{i, m}$ is the observed flow in month $m$ of year $i$, and $Q^{S}{ }_{i, m}$ is the simulated flow that has been generated with the meteorological input scenario in month $m$ of year $i$ using the initial condition in month $m$ of year $i$. The ratio for year $i$ is then applied to the scenario $i$ of a new forecasted ensemble for a forecasting time of month $m$, year $k$, i.e. $Q^{F}{ }_{i, m}(k)$ with the meteorological input scenario in month $m$ of year $i$ using the initial condition in month $m$ of year $k$.

$$
Q_{i, m}^{*}=\left(Q_{i, m}^{O} / Q_{i, m}^{S}\right) \times Q_{i, m}^{F}(k)
$$

The basic assumption of EBC is that ensemble scenarios have an identical bias if they come from the same meteorological input scenario even if their initial conditions are different.

\subsubsection{Optimal linear correction}

Theli (1971) proposed the optimal linear correction (OLC) method based on a linear regression model whose parameters are estimated from true and simulated values. To apply the OLC, there should be a strong linear correlation between the observed and the simulated streamflows. Parameters $\alpha$ and $\beta$ in Equation (4) are estimated through the linear relationship between $Q^{O}{ }_{i, m}$ and $Q^{S}{ }_{i, m}$ of all scenarios in year $i$. Once the parameters are estimated, a revised streamflow scenario, $Q^{*}{ }_{i, m}$, can be calculated with the predicted streamflow scenario, $Q^{F}{ }_{i, m}$, as given in Equation (4).

$$
Q_{i, m}^{*}=\alpha+\beta Q_{i, m}^{F}
$$

\subsubsection{Quantile mapping}

When QM is applied in the post-processor, the CDFs of $F_{O}$ and $F_{S}$ estimated from historical streamflow, $Q^{O}{ }_{i, m}$, and the simulated streamflow, $Q^{S}{ }_{i, m}$, of all scenarios in year $i$ are used to map the predicted streamflow $Q^{F}{ }_{i, m}$ to the revised streamflow $Q^{*}{ }_{i, m}$, as shown in Equation (5).

$$
Q_{i, m}^{*}=F_{o}^{-1}\left(F_{S}\left(Q_{i, m}^{F}\right)\right)
$$

\section{Application}

\section{I. Study basin}

To evaluate the performance of the various preand post-processor methods, the Soyanggang dam (SDam) and the Chungju dam (C-Dam) basins in Korea were selected. Constructed from 1967 to 1973, S-Dam has the largest storage capability in the Republic of Korea, and is currently supplying 1.2 billion $\mathrm{m}^{3} /$ year of water to the Seoul metropolitan area. The basin area of the dam is $2703 \mathrm{~km}^{2}$, and the annual average rainfall and inflow are $1100 \mathrm{~mm}$ and $55.5 \mathrm{~m}^{3} / \mathrm{sec}$, respectively. With the second largest storage capability, the C-Dam was built from 1978 to 1985 , and its drainage area is $6648 \mathrm{~km}^{2}$. The annual average rainfall and 

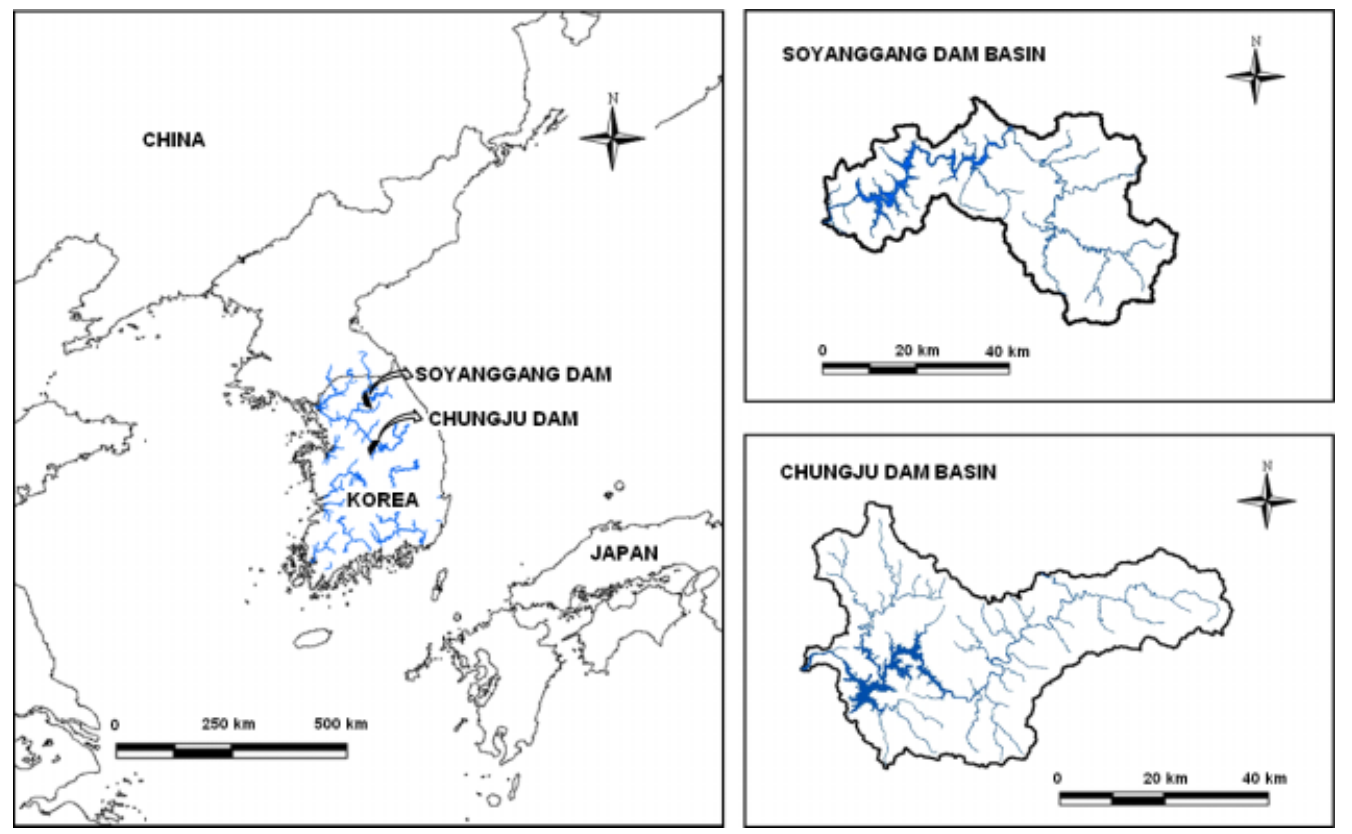

Figure 4. Soyanggang and Chungju dam basin in Korea.

Table I. The hit ratio of the KMA monthly categorical forecasts from 2000 to 2007.

\begin{tabular}{|c|c|c|c|c|c|c|c|c|c|}
\hline & \multicolumn{3}{|c|}{ Wet season } & \multicolumn{3}{|c|}{ Dry season } & \multicolumn{3}{|c|}{ Total } \\
\hline & AN_O & N_O & BN_O & AN_O & N_O & BN_O & AN_O & N_O & BN_O \\
\hline \multicolumn{10}{|c|}{ (a) Soyanggang dam } \\
\hline AN_F & $0.50(5)$ & $0.20(2)$ & $0.30(3)$ & $0.38(5)$ & $0.15(2)$ & $0.46(6)$ & $0.43(10)$ & $0.17(4)$ & $0.39(9)$ \\
\hline N_F & $0.45(9)$ & $0.30(6)$ & $0.25(5)$ & $0.29(10)$ & $0.35(12)$ & 0.35 (I2) & $0.35(19)$ & $0.33(18)$ & $0.31(17)$ \\
\hline BN_F & $0.50(1)$ & $0.00(0)$ & $0.50(1)$ & $0.24(4)$ & $0.18(3)$ & $0.59(10)$ & $0.26(5)$ & $0.16(3)$ & $0.58(11)$ \\
\hline \multicolumn{10}{|c|}{ (b) Chungju dam } \\
\hline AN_F & $0.50(5)$ & $0.30(3)$ & $0.20(2)$ & $0.23(3)$ & $0.31(4)$ & $0.46(6)$ & $0.35(8)$ & $0.30(7)$ & $0.35(8)$ \\
\hline N_F & $0.50(10)$ & $0.30(6)$ & $0.20(4)$ & $0.35(12)$ & $0.24(8)$ & $0.41(14)$ & $0.41(22)$ & $0.26(14)$ & $0.33(18)$ \\
\hline BN_F & $0.50(1)$ & $0.50(1)$ & $0.00(0)$ & $0.24(4)$ & $0.29(5)$ & $0.47(8)$ & $0.26(5)$ & $0.32(6)$ & $0.42(8)$ \\
\hline
\end{tabular}

inflow are $1197.6 \mathrm{~mm}$ and $154.5 \mathrm{~m}^{3} / \mathrm{sec}$, respectively (Figure 4). The main feature of the Korean hydrology is that two-third of the annual precipitation occurs only for the 3-month wet season from July, whereas only approximately $400 \mathrm{~mm}$ is available for the remaining 9-month dry season.

\subsection{Application of the categorical forecast}

\subsection{Evaluation of the climate forecast} and hydrologic model

As mentioned earlier, this study used the monthly categorical forecast of the KMA for the pre-processor. The categorical forecast, issued three times per month, yields the most probable temperature and rainfall range without probability. The three possible ranges are above-normal (AN), normal (N), and below-normal (BN) ranges. The accuracy of the historical KMA forecasts was evaluated with the hit ratio for a verification period from 2000 to 2007 .

Table I shows the resulting hit ratio for the tested sites. Note that the hit ratio of the naïve forecast is 0.33 because there are three categories. Among the three categories, the $\mathrm{BN}$ range had the highest hit ratio (0.58 and 0.42 ) and the AN range also performed reasonably well. Although the AN predictions are superior to the other ranges in the wet season, BN conversely shows the highest hit ratio for the dry season.

Estimated from the categorical forecast using CPM, the probabilistic climate forecasts from 2004 to 2007 were evaluated for the verification method with a ranked probability score (RPS), commonly used in probabilistic predictions.

In Table II, the meteorological ensemble of historical observations serves as the original input of ESP, and the probabilistic climate forecast applied through the pre-processors is the information used to adjust those observations. Table II shows that the probabilistic forecast can reflect the true climate condition more accurately than the meteorological ensemble during the wet season. However, the RPS of the probabilistic forecast during the dry season is conversely larger than the meteorological ensemble, especially at C-Dam. 
Table II. RPS of the meteorological ensemble and the probabilistic climate forecasts estimated from the KMA monthly categorical forecast from 2004 to 2007.

\begin{tabular}{lllll}
\hline & & $\begin{array}{c}\text { Wet } \\
\text { season }\end{array}$ & $\begin{array}{c}\text { Dry } \\
\text { season }\end{array}$ & Total \\
\hline Chugnju & Probabilistic climate forecast & $\mathbf{0 . 3 7 0}^{\dagger}$ & 0.419 & 0.403 \\
& Meteorological ensemble & 0.405 & $\mathbf{0 . 4 0 5}$ & 0.405 \\
& Reduction ratio* & $8.7 \%$ & $-3.5 \%$ & $0.5 \%$ \\
Soyanggang & Probabilistic climate forecast & $\mathbf{0 . 4 2 4}$ & 0.420 & 0.421 \\
& Meteorological ensemble & 0.455 & $\mathbf{0 . 4 1 8}$ & 0.430 \\
& Reduction ratio* & $6.7 \%$ & $-0.6 \%$ & $1.9 \%$ \\
\hline
\end{tabular}

* Reduction ratio $=$ (RPS of meteorological ensemble - RPS of probabilistic climate forecast)/RPS of meteorological ensemble.

$\dagger$ Superior RPS results between the probabilistic climate forecast and meteorological ensemble for each season.

The uncertainty in the hydrological model was also quantified. In this study, the TANK hydrologic model was used for ESP. Developed by Sugawara (1967), TANK is a conceptual rainfall runoff model composed of 12 parameters. To evaluate the performance of TANK compared with the observed inflows at S-Dam and C-Dam from 2004 to 2007, we used the RBias (Relative-Bias) and R-RMSE (Relative-RMSE), which are calculated as the bias and RMSE divided by the average of the observed streamflow, respectively. Table III shows that the uncertainty (R-RMSE) in the dry season is much greater than the uncertainty in the wet season, and the systematic error (R-Bias) is almost negligible at S-Dam during the wet season.

\subsubsection{Employing pre- and post-processors for ESP}

The monthly observed inflows at S-Dam and C-Dam from 2004 to 2007 were used for verification, and the resulting RPS values for various pre- and postprocessors are shown in Table IV. The 'No pre' row and the 'No post' column in Table IV represents a simple ESP without any pre- and post-processors. Those four cells (called ESP0) of intersection between 'No pre' and 'No post' have the values of 0.431 and 0.847 for S-Dam and 0.504 and 0.729 for C-Dam. The ESP is more accurate for the wet season than the dry season in both basins. This result would be strongly affected by the performance of the hydrologic model verified in Table III showing that the R-Bias and RRMSE are much smaller in wet season than in dry season.

From the above four ESP0 cells, one can move either horizontally or vertically. For both basins, the horizontal move lowers the RPS more than the vertical move, which implies that the use of the post-processor is more effective in improving the accuracy of the noprocessor ESP than that of the pre-processor. The table also demonstrates that the post-processor can be more effective for the tested basins in the dry season than in the wet season. The values of the four cells in the 'No pre' raw data for C-Dam (i.e. 0.504, 0.495, 0.414 and 0.484 ) are generally higher than the corresponding values below those cells except for 0.414 in the QM column, which indicates that the climate information used with the pre-processor was valuable for this basin during the wet season.

Without any processors, S-Dam produces more accurate ESP than C-Dam (i.e. 0.431 and 0.504, respectively), but the ESP accuracy of C-Dam becomes higher than that of S-Dam with an effective combination of the pre- and post-processors (i.e. 0.392 and 0.421 , respectively, using the QM pre-processor and the EBC post-processor). From Table II, we

Table III. R-Bias and R-RMSE of the simulated inflow using the TANK model from 2004 to 2007.

\begin{tabular}{|c|c|c|c|c|c|c|}
\hline & \multicolumn{2}{|c|}{ Wet season } & \multicolumn{2}{|c|}{ Dry season } & \multicolumn{2}{|c|}{ Total } \\
\hline & Soyanggang & Chungju & Soyanggang & Chungju & Soyanggang & Chungju \\
\hline R-bias & 0.045 & 0.087 & 0.463 & 0.310 & 0.162 & 0.142 \\
\hline R-RMSE & 0.391 & 0.350 & 0.711 & 0.736 & 0.608 & 0.568 \\
\hline
\end{tabular}

Table IV. RPS of monthly ESP with the pre- and post-processors from 2004 to 2007.

\begin{tabular}{|c|c|c|c|c|c|c|c|c|c|c|}
\hline & & & \multicolumn{8}{|c|}{ ESP post } \\
\hline & & & \multicolumn{4}{|c|}{ Soyanggang dam } & \multicolumn{4}{|c|}{ Chungju dam } \\
\hline & & & No post & EBC & QM & OLC & No post & EBC & QM & OLC \\
\hline ESP & Wet season & No Pre & 0.431 & 0.435 & 0.424 & 0.426 & 0.504 & 0.495 & 0.414 & 0.484 \\
\hline \multirow[t]{7}{*}{ Pre } & & QM & 0.446 & 0.464 & 0.433 & $0.44 I$ & 0.432 & 0.398 & 0.439 & 0.408 \\
\hline & & SS & 0.437 & 0.458 & $0.421^{*}$ & 0.429 & 0.437 & 0.393 & $0.392^{*}$ & 0.395 \\
\hline & & PR & 0.491 & 0.485 & 0.470 & 0.449 & 0.474 & 0.451 & 0.437 & 0.446 \\
\hline & Dry season & No pre & 0.847 & $0.414^{*}$ & 0.507 & 0.525 & 0.729 & 0.476 & $0.455^{*}$ & 0.575 \\
\hline & & QM & 0.813 & 0.418 & 0.501 & 0.550 & 0.649 & 0.502 & 0.512 & 0.56 \\
\hline & & SS & 0.828 & 0.456 & 0.540 & 0.589 & 0.713 & 0.515 & 0.509 & 0.611 \\
\hline & & PR & 0.822 & 0.413 & 0.526 & 0.511 & 0.713 & 0.458 & 0.457 & 0.546 \\
\hline
\end{tabular}

* Superior RPS results among the various combinations of the pre- and post-processors for each season and basin. 
Table V. R-Bias and R-RMSE of the I0-day SNURCM precipitation forecasts from 2004 to 2007.

\begin{tabular}{|c|c|c|c|c|c|c|}
\hline & \multicolumn{2}{|c|}{ Wet season } & \multicolumn{2}{|c|}{ Dry season } & \multicolumn{2}{|c|}{ Total } \\
\hline & Soyanggang & Chungju & Soyanggang & Chungju & Soyanggang & Chungju \\
\hline $\begin{array}{l}\text { R-bias } \\
\text { R-RMSE }\end{array}$ & $\begin{array}{r}-0.279 \\
1.383\end{array}$ & $\begin{array}{r}-0.263 \\
1.184\end{array}$ & $\begin{array}{l}0.419 \\
2.356\end{array}$ & $\begin{array}{l}0.333 \\
1.951\end{array}$ & $\begin{array}{r}-0.045 \\
2.053\end{array}$ & $\begin{array}{r}-0.065 \\
\mid .751\end{array}$ \\
\hline
\end{tabular}

Table VI. RPS of the I0-day ESP with the pre- and post-processors from 2004 to 2007.

\begin{tabular}{|c|c|c|c|c|c|c|c|c|c|c|}
\hline & & & \multicolumn{8}{|c|}{ ESP post } \\
\hline & & & \multicolumn{4}{|c|}{ Soyanggang dam } & \multicolumn{4}{|c|}{ Chungju dam } \\
\hline & & & No post & EBC & QM & OLC & No post & EBC & QM & OLC \\
\hline ESP & Wet season & No pre & 0.413 & 0.387 & 0.383 & 0.392 & 0.282 & 0.266 & 0.270 & 0.291 \\
\hline \multirow[t]{7}{*}{ Pre } & & QM & 0.406 & $0.38 I^{*}$ & 0.385 & 0.386 & 0.271 & 0.256 & 0.271 & 0.284 \\
\hline & & SS & 0.402 & 0.389 & 0.391 & 0.391 & 0.250 & $0.243^{*}$ & 0.255 & 0.273 \\
\hline & & PR & 0.424 & 0.393 & 0.393 & 0.404 & 0.294 & 0.278 & 0.280 & 0.306 \\
\hline & Dry season & No pre & 0.834 & 0.428 & 0.571 & 0.631 & 0.661 & 0.520 & 0.513 & 0.552 \\
\hline & & QM & 0.841 & 0.443 & 0.581 & 0.645 & 0.685 & 0.550 & 0.535 & 0.574 \\
\hline & & SS & 0.850 & 0.441 & 0.581 & 0.644 & 0.688 & 0.545 & 0.538 & 0.573 \\
\hline & & PR & 0.833 & $0.425^{*}$ & 0.567 & 0.627 & 0.652 & 0.514 & $0.507^{*}$ & 0.545 \\
\hline
\end{tabular}

* Superior RPS results among the various combinations of the pre- and post-processors for each season and basin.

could understand that this occurs because the climate forecast for C-Dam in the wet season (0.370 in Table II) is more accurate than that of S-Dam $(0.424$ in Table II). That is, when the systematic error of the hydrologic model is effectively diminished by the post-processor, the accuracy of the probabilistic climate forecast used in the pre-processor mainly contributes to the ESP accuracy, almost irrespective of the initial ESP0 accuracy without the pre- and postprocessor.

Before finding out which combination of the preand post-processors can provide more accurate forecasts for the applied basins, we need to make note of one more point. A good processor can be underestimated when other kinds of significant uncertainties that cannot be reduced by that processor alone dominate. The smallest RPS among the three methods used for C-Dam during the wet season occurs when there is no post-processor and QM is used as the pre-processor. However, when the hydrological bias in ESP has been reduced by a post-processor, it is shown in Table IV that SS produced a smaller RPS than QM. Therefore, it is recommended to apply and evaluate a pre-processor when the inherent hydrological bias is removed or at least reduced.

For the wet season, the combination of $\mathrm{SS}$ as the pre-processor and $\mathrm{EBC}$ or QM as the postprocessors maximizes the accuracy of ESP for both basins. For the dry season, the use of EBC or QM alone as the post-processor is sufficient because the hydrological uncertainty is considerably larger than the meteorological uncertainty during the dry season.

\subsection{Application of the deterministic forecast}

In addition to the use of the monthly categorical forecast, this study also applied the 10-day ahead deterministic forecast into the 10 day ESP system for the same test basins. The deterministic forecasts for this study were taken from the 10 day precipitation forecast from the Seoul National University Regional Climate Model (SNURCM). Developed by the project named Eco-Technopia 21 in Korea, SNURCM provides a forecast by considering the detailed topography based on the boundary conditions from the Community Climate System Model (CCSM) maintained by the National Center for Atmospheric Research.

First, the SNURCM precipitation forecasts were evaluated for a verification period from 2004 to 2007. Table $\mathrm{V}$ shows that both R-Bias and R-RMSE are smaller in the wet season and especially at C-Dam. Each 10-day deterministic forecast was then converted to a probabilistic forecast using DPC, and finally incorporated into the 10-day ESP system through using the three pre-processors. Table VI shows the resulting accuracy of the 10-day ESP system with RPS. Its performance was very similar to that of the monthly ESP system: using a pre-processor is more effective in the wet season than the dry season. This reflects the accuracy of the climate forecast shown in Table V. Among the three post-processors, EBC generally outperforms the others. However, the best combination of pre- and post-processors for the 10-day ESP is different compared from the best combination of the monthly ESP system. For S-Dam, the combination of QM and EBC performs best in the wet season and the combination of PR and EBC performs best in the dry season. For C-Dam, the 
combination of SS and EBC performs best in the wet season and the combination of PR and QM performs best in the dry season.

\subsection{Discussion}

The results shown in the previous sections are based on the present case study. In this study, however, some features of the tested pre- and post-processors that may be applicable to other case studies were found. First, care must be taken when QM and PR are used for high values because they are based on a continuous probabilistic distribution that is often very sensitive to a small change in probability in its tail. This is why QM and PR did not perform well during the Korean basin's wet season. SS performed consistently, however, because it is based on the discretized values of a continuous PDF. Second, EBC can be recommended as the best post-processor in general as a result of this study, especially when compared with OLC. This is because EBC emphasizes the model error in the specific year being considered, where OLC adjusts the model error using the flow amount based on the average error over the entire year. In other words, the temporal structure of the model error is very important in the ESP study. Finally, QM generally performs well for both pre- and postprocessors if it correctly estimates the quantiles of the variable being considered, which is the case here.

\section{Conclusions}

- In general, use of the post-processor method more effectively reduced the uncertainty of the noprocessor ESP than use of the pre-processor, especially in the dry season.

- QM generally performs well for both pre- and postprocessors if it estimates quantiles of a variable being considered correctly but a care must be taken when QM is used for high values. However, EBC generally performs best among the tested post-processors.

- A good pre-processor should be used together with a good post-processor that effectively removes or at least reduces the hydrological model uncertainty.
In this study, we attempted to present the best combination of pre- and post-processors but drawing a general conclusion would be still premature because of the limited lengths of the observations and the forecasts. Therefore, the present conclusion should be further evaluated with more case studies and more application periods.

\section{Acknowledgements}

This study has been financially supported by the Ministry of Land, Transport and Maritime Affairs under the project entitled 'Probabilistic Streamflow Prediction using Ensemble Model' and 'the Construction Technology Innovation Program (08Tech-Innovation-F01)' through the Research Center of Flood Defense Technology for Next Generation in Korea Institute of Construction and Transportation Technology Evaluation and Planning.

\section{References}

Clark M, Gangopadhyay S, Hay L, Rajagopalan B, Wilby R. 2004. The Schaake shuffle: A method for reconstructing space-time variability in forecasted precipitation and temperature fields. Journal of Hydrometeorology 5: 243-262.

Day GN. 1985. Ensemble streamflow forecasting using NWSRFS. Journal of Water Resources Planning and Management 111: 147-170.

Kang BS, Rieu SY, Ko IH. 2007. Long range probabilistic prediction using precipitation outlook weighted ensemble. Korean Society of Civil Engineers 27: 183-191.

Schaake J, Demargne J, Hartman R, Mullusky M, Welles E, Wu L, Herr H, Fan X, Seo D-J. 2007. Precipitation and temperature ensemble forecasts from single-value forecasts. Hydrology and Earth System Sciences Discussions 4: 655-717.

Seo D-J, Herr HD, Schaake JC. 2006. A statistical post-processor for accounting of hydrologic uncertainty in short-range ensemble streamflow prediction. Hydrology and Earth System Sciences Discussions 3: 1987-2035.

Shi X, Wood AW, Lettenmaier DP. 2008. How essential is hydrologic model calibration to seasonal streamflow forecasting. Journal of Hydrometeorology 9: 1350-1363.

Smith JA, Day GN, Kane MD. 1992. Nonparametric framework for long-range streamflow forecasting. Journal of Water Resources Planning and Management ASCE 118: 82-92.

Stedinger JR, Kim Y-O. 2002. Updating ensemble probabilities based on climate forecasts. Conference on Water Resources Planning and Management and Symposium on Managing the Extremes Floods and Droughts, EWRI, ASCE, Roanoke, VA.

Sugawara M. 1967. On the analysis of runoff structure about several Japanese rivers. Japanese Journal of Geophysics 2(4).

Theil H. 1971. Applied Economic Forecasting. North Holland Publishing Company: Amsterdam, Netherlands. 